\title{
Mediation of the effects of living in extremely poor neighborhoods by health insurance: breast cancer care and survival in California, 1996 to 2011
}

Kevin M Gorey ${ }^{1 *}$, Isaac N Luginaah² ${ }^{2}$ Eric J Holowaty ${ }^{3}$, Guangyong Zou ${ }^{4}$, Caroline Hamm ${ }^{5}$ and Madhan K Balagurusamy ${ }^{6}$

\begin{abstract}
Background: We examined the mediating effect of health insurance on poverty-breast cancer care and survival relationships and the moderating effect of poverty on health insurance-breast cancer care and survival relationships in California.

Methods: Registry data for 6,300 women with breast cancer diagnosed between 1996 and 2000 and followed until 2011 on stage at diagnosis, surgeries, adjuvant treatments and survival were analyzed. Socioeconomic data were obtained for residences from the 2000 census to categorize neighborhoods: high poverty (30\% or more poor), middle poverty (5\%-29\% poor) and low poverty (less than 5\% poor). Primary payers or health insurers were Medicaid, Medicare, private or uninsured.
\end{abstract}

Results: Evidence of survival mediation was observed for women with node negative breast cancer. The apparent effect of poverty disappeared in the presence of Medicare or private health insurance. Women who were so insured were advantaged on 8-year survival compared to the uninsured or those insured by Medicaid $(\mathrm{OR}=1.89)$. Evidence of payer moderation by poverty was also observed for women with node negative breast cancer. The survival advantaging effect of Medicare or private insurance was stronger in low poverty $(O R=1.81)$ than it was in middle poverty $(\mathrm{OR}=1.57)$ or in high poverty neighborhoods $(\mathrm{OR}=1.16)$. This same pattern of mediated and moderated effects was also observed for early stage at diagnosis, shorter waits for adjuvant radiation therapy and for the receipt of sentinel lymph node biopsies. These findings are consistent with the theory that more facilitative social and economic capital is available in low poverty neighborhoods, where women with breast cancer may be better able to absorb the indirect and direct, but uncovered, costs of care. As for treatments, main protective effects as well as moderator effects indicative of protection, particularly in high poverty neighborhoods were observed for women with private health insurance.

Conclusions: America's multi-tiered health insurance system mediates the quality of breast cancer care. The system is inequitable and unjust as it advantages the well insured and the well to do. Recent health care reforms ought to be enacted in ways that are consistent with their federal legislative intent, that high quality health care be truly available to all.

Keywords: Health insurance, Poverty, Breast cancer care, Survival, Social capital, Health care reform, California, United States

\footnotetext{
* Correspondence: gorey@uwindsor.ca

${ }^{1}$ School of Social Work, University of Windsor, 401 Sunset Avenue, Windsor, Ontario N9B 3P4, Canada

Full list of author information is available at the end of the article
} 


\section{Background}

Studies of cancer care and survival in low-income areas of Canada and the United States have consistently observed Canadian advantages over the past generation [1-5]. More inclusive health insurance in Canada has been advanced as the most plausible explanation. These studies were ecological with respect to the measurement of socioeconomic status (SES). They used census tract data to define low-income neighborhoods, however, their lowest income areas typically only ranged from $10 \%$ to $20 \%$ poor. So they had limited power to study cancer care among "the truly disadvantaged" that live in America's poorest neighborhoods where $30 \%$ to $40 \%$ or more of the households have incomes below the poverty line [6-8].

Recent analyses have suggested several reasons for the importance of investigating breast cancer care in such vulnerable places $[2,5]$. First, breast cancer is the most common cancer among American women and its prognosis is typically excellent with early diagnosis and treatment $[9,10]$. Second, a direct association between income and breast cancer survival has been consistently observed [2,11]. Third, as increasingly effective screens and treatments have proliferated evidence has mounted that the best care is more accessible to women of higher SES [3-5,12-17]. Fourth, being underinsured has also been associated with less than optimum care [18-24]. These US studies have also for the most part had insufficient samples and statistical power to study the quality of breast cancer care among those at greatest risk of not receiving it; the very poor and the inadequately insured.

\section{Combined effects of being underinsured and living in extremely poor neighborhoods}

International studies have allowed us to develop the theory that SES-cancer care relationships are probably mediated by health insurance [2,25-27]. It is also probable that within the multi-payer US health care system the effects of various health insurers interact with other resources in ways not yet studied. However, thus far this field has primarily focused on advancing knowledge about main effects. More complex hypotheses about mediation and moderation effects have, for the most part, not been formally tested [17]. A recent study of ours may have been the first to test such effects in American cancer care [27]. We found evidence that having health insurance significantly mediated or buffered the povertysurvival relationship for women with colon cancer, but not for men. We also found that poverty moderated the health insurance-survival relationship, again only for women. The advantaging effects of all health insurers, public and private, were significantly larger for women in low-poverty neighborhoods. The present study will examine these relationships among women with breast cancer.

\section{Health insurance in transaction with social capital}

Our SES-health insurance-cancer care mediation theory aims to model care gaps in America's most vulnerable communities. Its foundation is William Julius Wilson's work in 1960s Chicago's high poverty neighborhoods and Paul Jargowsky and Mary Jo Bane's research in America's extremely poor neighborhoods in the 1970s and 80s [6-8]. Together they described places of prevalent demographic vulnerability that were particularly distressed for their lack of social and economic capital. Medicaid and Medicare, the government's, respective, mean-tested and entitlement, health insurance programs were specifically designed to mediate the effects of such impoverishment. But SES and public and private health insurance probably interact in important ways across America's economic divide. A recent survey demonstrated how SES and health insurance can interact in the lives of people with cancer. It surveyed mostly insured people with colon cancer and found that more than a third of them suffered one or more significant financial hardships as a result of their treatment [28]. Moreover, more than one of every ten of them reported coverage denials by their insurers with consequent inabilities to adhere to prescribed treatment regimes. And low-income households were much more likely to be so impacted. It seems that the effectiveness of various health insurance programs may be significantly moderated by other resources. Those most likely to be disadvantaged are the poor. It is they who are the least likely to be able to bare the indirect (time lost from work, child care and travel) and direct, but uncovered costs (deductibles, co-insurances and co-payments) of contemporary cancer care in America [29].

\section{Hypotheses}

The objective of this study was to evaluate the distinct mediating and moderating effects of SES and health insurance on cancer care in extremely poor communities in the US. We hypothesized that the inverse povertybreast cancer survival relationship would be significantly, if not completely, mediated or explained by the intermediate effect of having health insurance (private, Medicare or Medicaid). We also hypothesized a health insurance by poverty interaction, that is, that the direct insurancebreast cancer survival relationship would be significantly moderated by poverty (all types of health insurance would be less effective in high poverty neighborhoods) [30,31]. We then explored similar hypotheses across breast cancer care from diagnoses to adjuvant or palliative therapies.

\section{Methods}

The sampling frame was the California cancer registry (CCR) which validly monitors the most populous US state [32-34]. California seems an appropriate place for this study as its population in high poverty 
neighborhoods nearly doubled between 1990 and 2000 while the nation's population in high poverty neighborhoods decreased significantly $[35,36]$. Registry data was obtained for 6,300 women with invasive breast cancer diagnosed between 1996 and 2000 [37] and followed until 2011. Cases were randomly selected from three geographic place strata (2,100 cases each): megalopolises (greater San Diego, the San Francisco Bay area and greater Los Angeles), smaller metropolitan areas (Salinas, Modesto, Stockton, Bakersfield and Fresno) and rural places with population densities less than 400 per $\mathrm{km}^{2}$ [38-40]. This study was powered to detect rate differences of $5 \%$ between 3 geographic place and 3 socioeconomic strata with $80 \%$ power at a 2 -tailed significance level of $5 \%$ $[41,42]$.

\section{Variables}

\section{Poverty and payers}

We first linked breast cancer patients to the US (2000) census by their residential census tracts [38]. Next, to model our poverty measure after those that have been the most validated, we defined the following neighborhoods: high poverty ( $30 \%$ or more of households poor), middle poverty (5\%-29\% poor) and low poverty (less than $5 \%$ poor) [2,5-8,43-45]. Socioeconomic distributions of our sample's poverty tertiles are displayed in Table 1 . The prevalence of poverty in the typical high poverty neighborhood was $37 \%$, just about mid-point between Wilson's (30\%) and Jargowsky and Bane's (40\%) criterion definitions of high poverty. The typical annual household income of $\$ 23,275$ in such high poverty neighborhoods in 2000 seems a face valid definition of an extremely poor household. Most of this field's heretofore low-income or "poor" samples would have fell into this study's middle poverty group.

Health insurance status, defined as the primary source of payment to the hospital or primary payer, was determined from medical records during the initial course of treatment. It was categorized as uninsured, Medicaid, Medicare (with or without any supplementation or any other non-means-tested governmental payer) or private insurer (any commercial managed care corporation or fee-for-service provider). The two key study variables of

Table 1 Prevalence of poor households in breast cancer patients' neighborhoods: California, 2000

\begin{tabular}{llcc}
\hline \multirow{2}{*}{$\begin{array}{l}\text { Neighborhood } \\
\text { poverty }\end{array}$} & \multicolumn{2}{c}{$\begin{array}{c}\text { Prevalence (\%) of households } \\
\text { Living in poverty }\end{array}$} & \\
\cline { 2 - 3 } & \multicolumn{1}{c}{ Range } & Median & Income, ${ }^{\text {a }} \mathbf{\$}$ \\
\hline Low & $0.00-4.99$ & 3.36 & 74,000 \\
Middle & $5.00-29.76$ & 11.40 & 44,200 \\
High & $30.09-100.00$ & 36.84 & 23,275 \\
\hline
\end{tabular}

Note. Neighborhood income derived from US Census data [38].

${ }^{a}$ Census tract median annual household income. neighborhood poverty and primary payer are crosstabulated in Table 2. Women with breast cancer living in high poverty California neighborhoods were one and a half times as likely to be uninsured, more than twelve times as likely to be primarily insured by Medicaid, but only two-thirds as likely to be covered by a private insurer as were their counterparts in low poverty neighborhoods.

\section{Breast cancer care}

Variables from hospital and physicians' office charts and clinic reports were coded by the CCR $[46,47]$. These were stage of disease at diagnosis (node negative, node positive or distally metastasized), receipt of initial surgery, type of surgery (lumpectomy or mastectomy), receipt of adjuvant radiation therapy, chemotherapy or hormone therapy, wait times from diagnosis to initial surgery and from surgery to adjuvant therapies, receipt of breast reconstruction surgery (post-mastectomy) and survival time from diagnosis to death or last follow-up at 10 years. All of these variables had less than $5 \%$ missing data. Tumor size (88.2\%), histological grade (85.1\%) and hormone receptor statuses (estrogen and progesterone receptors, 93.3\%) were also coded for the vast majority of the sample. Various long wait criteria that may be associated with recurrences, metastases or shorter survival were explored [3,48-52].

Table 2 Primary payers among women with breast cancer in low, middle and high poverty neighborhoods: age-adjusted prevalence estimates and standardized prevalence ratios

\begin{tabular}{|c|c|c|c|c|}
\hline \multicolumn{5}{|l|}{ Neighborhood poverty } \\
\hline By primary payer & Sample & Prevalence & PR & $(95 \% \mathrm{Cl})$ \\
\hline \multicolumn{5}{|l|}{ Low Poverty } \\
\hline Uninsured & 183 & .086 & 1.00 & \\
\hline Medicaid & 25 & .012 & 1.00 & \\
\hline Medicare & 525 & .263 & 1.00 & \\
\hline Private & 1,367 & .638 & 1.00 & \\
\hline \multicolumn{5}{|l|}{ Middle Poverty } \\
\hline Uninsured & 226 & .108 & 1.26 & $(1.04,1.52)$ \\
\hline Medicaid & 130 & .062 & 5.17 & $(3.60,7.42)$ \\
\hline Medicare & 577 & .275 & 1.05 & $(0.94,1.17)$ \\
\hline Private & 1,167 & .555 & 0.87 & $(0.83,0.91)$ \\
\hline \multicolumn{5}{|l|}{ High Poverty } \\
\hline Uninsured & 276 & .133 & 1.55 & $(1.30,1.85)$ \\
\hline Medicaid & 310 & .151 & 12.58 & $(9.28,17.05)$ \\
\hline Medicare & 672 & .307 & 1.17 & $(1.06,1.29)$ \\
\hline Private & 842 & .408 & 0.64 & $(0.60,0.68)$ \\
\hline
\end{tabular}


Descriptive profiles of the breast cancer patients in our sample are displayed in Table 3. As planned, diverse geographic places, large urban to rural, and types of neighborhoods, high poverty to relatively affluent, were represented. Demographically, our age-diverse sample of younger working-age women (57.6\%) and older women of retirement age (42.3\%) seems consistent with expectations. More than half of them were primarily covered by a commercial, private insurer (53.6\%). Given our oversampling of very poor neighborhoods, the relatively low representation of the uninsured (10.9\%) may seem surprising. Note though that most initial breast cancer care takes place in hospitals where social workers work to connect uninsured patients to any additional resources, typically Medicaid or Medicare, for which they are qualified by virtue of being poor, older or disabled.

\section{Statistical analyses}

We used logistic regression models to test hypotheses about the mediating and moderating effects of poverty and payers in predicting binary (survived or not) allcause breast cancer survival [53-55]. Odds ratios (ORs) and $95 \%$ confidence intervals (CIs) were estimated. Survival outcomes that were best predicted by main poverty and payer effects and their interactions were analyzed and reported: 8-year for node negative, 5-year for node positive and 3-year for distally metastasized disease (Hosmer-Lemeshow test $[55,56])$. ORs estimate the relative predictive weights of main and interacting effects. However, under the circumstances of this study, where both social exposures and key outcomes are common, ORs probably overestimate rate ratios (RRs) [57]. So we provided accompanying practical assessments more germane perhaps to clinical and policy significance. Rates were directly adjusted for age and other relevant covariates that significantly and substantially confounded poverty- or payer-breast cancer care relationships, using this study's sample as the standard and reported as rates per 100 cases or percentages. Then we used standardized RRs for between-group comparisons, with 95\% CIs derived from the Mantel-Haenszel $\chi^{2}$ test $[58,59]$. Statistical model tests (ORs with 95\% CIs) are presented in Tables 4 ,5, 6, 7 and 8 with accompanying "real world" practical significance indices (RRs with $95 \% \mathrm{CIs}$ ) reported as interpretive adjuncts in the text. Because some of our subsample-based hypotheses could be deemed exploratory we also reported as approaching significance any finding that met the more liberal statistical criterion of $p<.10$.

\section{Results}

Interacting effects of poverty and health insurance on breast cancer survival

Regression models for node negative, node positive and metastasized breast cancer survival are displayed in
Table 4. Substantial support for both mediation and moderation hypotheses was observed for node negative disease. The top left column shows significant main effects of poverty $(\mathrm{OR}=0.54)$ and payer $(\mathrm{OR}=1.57)$ when these factors entered regression models alone. Moving down to the full model, consistent with mediation, in the presence of payer the effect of poverty disappeared and the effect of adequate payers was strengthened $(\mathrm{OR}=1.89)$. The 8-year survival rate among those insured privately or by Medicare $(75.5 \%)$ was nine percent greater than that of the uninsured or those covered by Medicaid (69.0\%, RR $=1.09,95 \% \mathrm{CI}$ $1.03,1.15)$.

The hypothesis that the health insurance-survival relationship would be moderated by poverty was also supported for women with node negative disease. The statistical interaction's practical effect moderation is depicted in the bottom of Table 4. Having adequate health insurance seemed much more effective in low poverty $(\mathrm{OR}=1.81,95 \% \mathrm{CI} 1.11,2.95)$ than in high poverty neighborhoods, where Medicare or private insurance did not seem any more effective than having Medicaid or being uninsured $(\mathrm{OR}=1.16,95 \%$ CI 0.82, 1.62). Consistent with social capital theory, the 8-year survival rate among women with node negative breast cancer who lived in low poverty neighborhoods and were primarily covered by Medicare or private insurers (79.9\%) was $13 \%$ greater than the survival rate among, otherwise similar, women primarily covered by Medicaid or who were uninsured $(70.8 \%, \mathrm{RR}=1.13,95 \%$ CI 1.02 , 1.25). A somewhat smaller payer gradient was observed in middle poverty neighborhoods $(75.5 \%$ vs $68.4 \%, \mathrm{RR}=$ $1.10,95 \%$ CI $1.01,1.20$ ) and no such gradient was observed in high poverty neighborhoods.

As for women with node positive and metastasized breast cancer, only significant main effects were observed. The practical 5-year survival effect of having Medicare or private coverage, versus Medicaid or none, for women with node positive disease was similar to that observed for women with node negative disease. Their respective survival rates were $73.6 \%$ and $66.2 \%$ ( $R R=$ $1.11,95 \%$ CI 1.03, 1.19). And having such coverage seems to have made a very large difference in the lives of women diagnosed with metastasized disease. Those who were adequately covered were much more likely to have survived for 3 years $(31.8 \%)$ than were the inadequately covered $(18.2 \%, \mathrm{RR}=1.75,95 \%$ CI $1.11,2.75)$.

\section{Interacting effects of poverty and health insurance on breast cancer diagnoses and treatments Stage at diagnosis}

Mediation and moderation hypothesis support was also found for early diagnosis of node negative disease. In the full regression model, the apparent effect of living in 
Table 3 Stratification, age, payer, staging, tumor and care characteristics of breast cancer patients diagnosed between 1996 and 2000 and followed until 2011

\begin{tabular}{|c|c|c|c|c|c|}
\hline & Sample & $\%$ & & Sample & $\%$ \\
\hline \multicolumn{6}{|c|}{ Stratification characteristics } \\
\hline Places & \multicolumn{5}{|c|}{ Poverty prevalence (\%) in neighborhoods } \\
\hline Large urban & 2,100 & 33.3 & $<5$ & 2,100 & 33.3 \\
\hline Smaller urban & 2,100 & 33.3 & $5-29$ & 2,100 & 33.3 \\
\hline Rural & 2,100 & 33.3 & $\geq 30$ & 2,100 & 33.3 \\
\hline \multicolumn{6}{|c|}{ Age and primary payer characteristics } \\
\hline Age at diagnosis, y & & & Primary payers & & \\
\hline $25-44$ & 904 & 14.3 & Private insurers & 3,376 & 53.6 \\
\hline $45-54$ & 1,386 & 22.0 & Medicare & 1,777 & 28.2 \\
\hline $55-64$ & 1,344 & 21.3 & Medicaid & 465 & 7.4 \\
\hline $65-74$ & 1,375 & 21.8 & Uninsured & 685 & 10.9 \\
\hline$\geq 75$ & 1,291 & 20.5 & & & \\
\hline \multicolumn{6}{|c|}{ Staging characteristics at diagnosis } \\
\hline Summary stage & & & Size of tumor, mm & & \\
\hline Local-regional & & & $<10$ & 975 & 17.5 \\
\hline Node negative & 4,035 & 66.2 & $10-19$ & 2,035 & 36.6 \\
\hline Node positive & 1,774 & 29.1 & $20-49$ & 2,035 & 36.6 \\
\hline Distally metastasized & 287 & 4.7 & $\geq 50$ & 512 & 9.2 \\
\hline Missing data & 204 & 3.2 & Missing data & 743 & 11.8 \\
\hline \multicolumn{6}{|c|}{ Tumor characteristics } \\
\hline Histological grade & & & Hormone receptor status & & \\
\hline I, well differentiated & 1,134 & 21.1 & Estrogen positive & 3,720 & 63.3 \\
\hline II, moderately & 2,211 & 41.2 & Progesterone positive & 3,189 & 54.3 \\
\hline III/IV, poorly & 2,019 & 37.6 & Either positive & 3,852 & 65.5 \\
\hline Missing data & 936 & 14.9 & Missing data & 422 & 6.7 \\
\hline \multicolumn{6}{|c|}{ Cancer care characteristics } \\
\hline Surgery received & & & Wait time from diagnosis & & \\
\hline Lumpectomy & 3,025 & 48.4 & $\leq 7$ & 2,699 & 45.8 \\
\hline Mastectomy & 2,876 & 46.0 & $8-30$ & 2,011 & 34.1 \\
\hline No surgery & 348 & 5.6 & $\geq 31$ & 1,187 & 20.1 \\
\hline Missing data & 51 & 0.8 & Missing data & 4 & 0.1 \\
\hline Received RT & 2,843 & 45.4 & Wait time after surgery $f$ & & \\
\hline \multirow[t]{5}{*}{ Missing data } & 44 & 0.7 & $\leq 60^{\mathrm{a}}$ & 1190 & 42.9 \\
\hline & & & $61-90$ & 451 & 16.3 \\
\hline & & & $91-180$ & 687 & 24.8 \\
\hline & & & $\geq 181$ & 446 & 16.1 \\
\hline & & & Missing data & 69 & 2.4 \\
\hline Received chemotherapy & 2,299 & 37.6 & \multicolumn{3}{|c|}{ Wait time after surgery for chemotherapy, $d$} \\
\hline \multirow[t]{5}{*}{ Missing data } & 185 & 2.9 & $\leq 30^{\mathrm{b}}$ & 891 & 40.7 \\
\hline & & & $31-60$ & 839 & 38.3 \\
\hline & & & $61-90$ & 273 & 12.4 \\
\hline & & & $\geq 91$ & 188 & 8.6 \\
\hline & & & Missing data & 108 & 4.7 \\
\hline
\end{tabular}


Table 3 Stratification, age, payer, staging, tumor and care characteristics of breast cancer patients diagnosed between 1996 and 2000 and followed until 2011 (Continued)

\begin{tabular}{|c|c|c|c|c|c|}
\hline Received HT & 2,115 & 34.7 & Wait time after surgery for $\mathrm{HT}, \mathrm{d}$ & & \\
\hline \multirow[t]{5}{*}{ Missing data } & 205 & 3.3 & $\leq 30^{c}$ & 701 & 34.7 \\
\hline & & & $31-90$ & 667 & 33.0 \\
\hline & & & $91-180$ & 408 & 20.2 \\
\hline & & & $\geq 181$ & 247 & 12.2 \\
\hline & & & Missing data & 92 & 4.3 \\
\hline
\end{tabular}

Note. $R T=$ radiation therapy, $H T=$ hormone therapy.

${ }^{a}$ A few (41) of these patients received RT prior to or without surgery.

${ }^{\mathrm{b}}$ One third $(32.4 \%)$ of these patients received chemotherapy prior to or without surgery.

c One fifth (18.5\%) of these patients received HT prior to or without surgery.

Table 4 Logistic regression main effects and interactions of neighborhood poverty and primary payer by breast cancer stage at diagnosis on survival

\begin{tabular}{|c|c|c|c|c|c|c|c|c|c|}
\hline \multirow[b]{3}{*}{ Predictor Variables } & \multicolumn{6}{|c|}{ Local-Regional } & \multicolumn{3}{|c|}{ Distally Metastasized } \\
\hline & \multicolumn{3}{|c|}{$\begin{array}{c}\text { Node negative disease } \\
8 \text { year survival }\end{array}$} & \multicolumn{3}{|c|}{$\begin{array}{c}\text { Node positive disease } \\
5 \text { year survival }\end{array}$} & \multicolumn{3}{|c|}{3 year survival } \\
\hline & Sample & OR & $(95 \% \mathrm{Cl})$ & Sample & OR & $(95 \% \mathrm{Cl})$ & Sample & OR & $(95 \% \mathrm{Cl})$ \\
\hline & \multicolumn{9}{|c|}{ Single predictor models } \\
\hline \multicolumn{10}{|l|}{ Neighborhood poverty } \\
\hline$<5 \%$ poor & 1,435 & 1.00 & & 561 & 1.00 & & 55 & 1.00 & \\
\hline $5-29 \%$ poor & 1,353 & 0.73 & $(0.61,0.88)$ & 574 & 0.70 & $(0.53,0.93)$ & 97 & 0.76 & $(0.37,1.59)$ \\
\hline$\geq 30 \%$ poor & 1,247 & 0.54 & $(0.45,0.65)$ & 639 & 0.41 & $(0.31,0.53)$ & 135 & 0.63 & $(0.31,1.29)$ \\
\hline \multicolumn{10}{|l|}{ Primary payer } \\
\hline Uninsured or Medicaid & 572 & 1.00 & & 382 & 1.00 & & 84 & 1.00 & \\
\hline \multirow[t]{2}{*}{ Medicare or private } & 3,463 & 1.57 & $(1.27,1.96)$ & 1,392 & 1.86 & $(1.44,2.41)$ & 203 & 2.51 & $(1.28,4.92)$ \\
\hline & \multicolumn{9}{|c|}{ Full models } \\
\hline \multicolumn{10}{|l|}{ Neighborhood poverty } \\
\hline$<5 \%$ poor & 1,435 & 1.00 & & 561 & 1.00 & & 55 & 1.00 & \\
\hline $5-29 \%$ poor & 1,353 & 0.92 & $(0.67,1.26)$ & 574 & 0.72 & $(0.55,0.96)$ & 97 & 0.79 & $(0.38,1.68)$ \\
\hline$\geq 30 \%$ poor & 1,247 & 0.84 & $(0.50,1.40)$ & 639 & 0.44 & $(0.34,0.58)$ & 135 & 0.76 & $(0.36,1.59)$ \\
\hline \multicolumn{10}{|l|}{ Primary payer } \\
\hline Uninsured or Medicaid & 572 & 1.00 & & 382 & 1.00 & & 84 & 1.00 & \\
\hline Medicare or private & 3,463 & 1.89 & $(1.26,2.84)$ & 1,392 & 1.59 & $(1.22,2.07)$ & 203 & 2.40 & $(1.21,4.79)$ \\
\hline \multirow[t]{4}{*}{ Poverty by payer } & 4,035 & $0.79^{*}$ & $(0.60,1.04)$ & 1,774 & $1.20^{\mathrm{a}}$ & $(0.85,1.69)$ & 287 & $1.17^{\mathrm{a}}$ & $(0.39,3.54)$ \\
\hline & \multicolumn{9}{|c|}{ Poverty by payer interaction among women with node negative disease on 8 year survival } \\
\hline & \multicolumn{3}{|c|}{$>30 \%$ poor } & \multicolumn{3}{|c|}{$5-29 \%$ poor } & \multicolumn{3}{|c|}{$<5 \%$ poor } \\
\hline & Sample & OR & $(95 \% \mathrm{Cl})$ & Sample & OR & $(95 \% \mathrm{Cl})$ & Sample & OR & $(95 \% \mathrm{Cl})$ \\
\hline
\end{tabular}

Primary payer

\begin{tabular}{|c|c|c|c|c|c|c|c|c|c|}
\hline Uninsured or Medicaid & 277 & 1.00 & & 186 & 1.00 & & 109 & 1.00 & \\
\hline Medicare or private & 970 & 1.16 & $(0.82,1.62)$ & 1,167 & 1.57 & $(1.09,2.27)$ & 1,326 & 1.81 & $(1.11,2.95)$ \\
\hline
\end{tabular}

Notes. $O R=$ odds ratio, $C l=$ confidence interval. All effects were age-adjusted across these categories: $25-44,45-54,55-64,65-74$ and 75 or older. After age, poverty and payer were accounted for, place (large urban, smaller urban or rural) and race/ethnicity (person of color [32.1\%] or non-Hispanic white) did not enter any of the full models. Bolded ORs were statistically significant at $p<.05$.

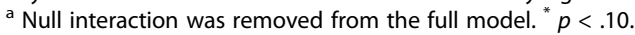


Table 5 Logistic regression main effects and interactions of neighborhood poverty and primary payer on breast cancer stage at diagnosis

\begin{tabular}{|c|c|c|c|c|c|c|}
\hline \multirow[b]{2}{*}{ Predictor Variables } & \multicolumn{3}{|c|}{ Node negative disease } & \multicolumn{3}{|c|}{ Tumor $<20 \mathrm{~mm}$} \\
\hline & Sample & OR & $(95 \% \mathrm{Cl})$ & Sample & OR & $(95 \% \mathrm{Cl})$ \\
\hline \multicolumn{7}{|c|}{ Single predictor models } \\
\hline \multicolumn{7}{|l|}{ Neighborhood poverty } \\
\hline$<5 \%$ poor & 1,833 & 1.00 & & 1,704 & 1.00 & \\
\hline $5-29 \%$ poor & 1,760 & 0.94 & $(0.81,1.08)$ & 1,639 & $0.88^{*}$ & $(0.75,1.02)$ \\
\hline$\geq 30 \%$ poor & 1,723 & 0.75 & $(0.65,0.88)$ & 1,575 & 0.61 & $(0.52,0.71)$ \\
\hline \multicolumn{7}{|l|}{ Primary payer } \\
\hline Uninsured or Medicaid & 888 & 1.00 & & 796 & 1.00 & \\
\hline Medicare or private & 4,428 & 1.42 & $(1.21,1.65)$ & 4,122 & 1.37 & $(1.16,1.61)$ \\
\hline \multicolumn{7}{|c|}{ Full models } \\
\hline \multicolumn{7}{|l|}{ Neighborhood poverty } \\
\hline$<5 \%$ poor & 1,833 & 1.00 & & 1,704 & 1.00 & \\
\hline $5-29 \%$ poor & 1,760 & 1.27 & $(0.88,1.84)$ & 1,639 & 0.89 & $(0.76,1.04)$ \\
\hline$\geq 30 \%$ poor & 1,723 & 1.06 & $(0.74,1.52)$ & 1,575 & 0.63 & $(0.54,0.73)$ \\
\hline \multicolumn{7}{|l|}{ Primary payer } \\
\hline Uninsured or Medicaid & 888 & 1.00 & & 796 & 1.00 & \\
\hline Medicare or private & 4,428 & 1.73 & $(1.23,2.44)$ & 4,122 & 1.23 & $(1.04,1.45)$ \\
\hline Poverty by payer & 5,316 & $0.72^{*}$ & $(0.49,1.06)$ & 4,918 & $1.16^{\mathrm{a}}$ & $(0.93,1.45)$ \\
\hline \multicolumn{7}{|c|}{ Poverty by payer interaction on node negative disease at diagnosis } \\
\hline & & $>5 \% \mathrm{p}$ & & & $<5 \% \mathrm{p}$ & \\
\hline Predictor Variables & Sample & $\mathrm{OR}$ & $(95 \% \mathrm{Cl})$ & Sample & OR & $(95 \% \mathrm{Cl})$ \\
\hline \multicolumn{7}{|l|}{ Primary payer } \\
\hline Uninsured or Medicaid & 730 & 1.00 & & 158 & 1.00 & \\
\hline Medicare or private & 2,753 & 1.28 & $(1.07,1.53)$ & 1,675 & 1.76 & $(1.25,2.48)$ \\
\hline
\end{tabular}

Notes. $\mathrm{OR}=$ odds ratio, $\mathrm{Cl}=$ confidence interval. All effects were age and grade-adjusted across these categories: 25-44, 45-54, 55-64, 65-74 and 75 or older; and well, moderately or poorly differentiated. Bolded ORs were statistically significant at $p<.05$.

${ }^{a}$ Null interaction was removed from the full model. ${ }^{*} p<.10$.

high poverty neighborhoods disappeared, whereas the effect of payer $(\mathrm{OR}=1.73)$ seemed strengthened and a poverty by payer interaction that approached statistical significance was detected (left column of Table 5). Evidence of this moderation of the effect of payer by poverty is depicted in the bottom of the table: low poverty $(\mathrm{OR}=1.76)$ and middle to high poverty neighborhoods $(\mathrm{OR}=1.28)$. In low poverty neighborhoods, women with Medicare or private health insurance were nearly $20 \%$ more likely $(69.1 \%)$ to have been diagnosed with node negative disease than were the uninsured or those covered by Medicaid $(58.3 \%, \mathrm{RR}=1.19,95 \%$ CI $1.05,1.34)$. Whereas the effectiveness of such health insurers was much smaller in middle to high poverty neighborhoods, where their respective early diagnosis rates were $65.3 \%$ and $61.5 \%(\mathrm{RR}=1.06,95 \% \mathrm{CI} 1.00,1.13)$. As for tumor size at diagnosis, only significant main effects of high poverty $(\mathrm{OR}=0.63)$ and payer $(\mathrm{OR}=1.23)$ were observed, and the practical size of the payer-tumor size relationship was similar to that of the payer-early stage relationship.

\section{Waits for care}

Wait times for initial surgery and adjuvant radiation therapy of local-regional breast cancers are displayed in Table 6. Two findings are consistent with mediation and moderation hypotheses. First, payer clearly mediated the poverty-wait time relationship among women who received radiation therapy without chemotherapy as the apparent effect of poverty disappeared in the presence of the highly significant effect of payer $(\mathrm{OR}=0.55$, middle column of Table 6). In fact, the rate of waiting for more than three months after surgery for radiation therapy among those covered by Medicare or private insurers (13.5\%) was less than half that of the uninsured or Medicaid covered $(28.6 \%, \mathrm{RR}=0.47,95 \%$ CI $0.36,0.61)$. Second, poverty seemed to moderate the payer-wait time relationship among women who received adjuvant radiation therapy with chemotherapy as there was an effect of payer that approached statistical significance in middle and low poverty neighborhoods ( $\mathrm{OR}=0.70, p<.10)$, but not in high poverty neighborhoods (bottom of Table 6). 
Table 6 Logistic regression main effects and interactions of neighborhood poverty and primary payer on wait times from diagnosis to surgery and from surgery to radiation therapy among women with local-regional breast cancer

\begin{tabular}{|c|c|c|c|c|c|c|c|c|c|}
\hline \multirow[b]{2}{*}{ Predictor variables } & \multicolumn{3}{|c|}{$\begin{array}{c}\text { Waited > } 60 \text { days } \\
\text { diagnosis to surgery }\end{array}$} & \multicolumn{3}{|c|}{$\begin{array}{c}\text { [No chemotherapy] } \\
\text { Waited }>90 \text { days } \\
\text { surgery to radiation therapy }\end{array}$} & \multicolumn{3}{|c|}{$\begin{array}{c}\text { [Had chemotherapy] } \\
\text { Waited }>210 \text { days } \\
\text { surgery to radiation therapy }\end{array}$} \\
\hline & Sample & OR & $(95 \% \mathrm{Cl})$ & Sample & OR & $(95 \% \mathrm{Cl})$ & Sample & OR & $(95 \% \mathrm{Cl})$ \\
\hline & \multicolumn{9}{|c|}{ Single predictor models } \\
\hline \multicolumn{10}{|l|}{ Neighborhood poverty } \\
\hline$<5 \%$ poor & 1,975 & 1.00 & & 640 & 1.00 & & 403 & 1.00 & \\
\hline $5-29 \%$ poor & 1,901 & 1.21 & $(0.92,1.59)$ & 507 & 1.06 & $(0.71,1.57)$ & 382 & 0.84 & $(0.59,1.18)$ \\
\hline$\geq 30 \%$ poor & 1,841 & 1.88 & $(1.46,2.43)$ & 382 & 1.50 & $(1.01,2.25)$ & 361 & 0.89 & $(0.63,1.26)$ \\
\hline \multicolumn{10}{|l|}{ Primary payer } \\
\hline Uninsured or Medicaid & 934 & 1.00 & & 224 & 1.00 & & 252 & 1.00 & \\
\hline Medicare or private & 4,783 & 0.61 & $(0.48,0.77)$ & 1,305 & 0.52 & $(0.35,0.77)$ & 894 & 0.87 & $(0.62,1.23)$ \\
\hline & \multicolumn{9}{|c|}{ Full models } \\
\hline \multicolumn{10}{|l|}{ Neighborhood poverty } \\
\hline$<5 \%$ poor & 1,975 & 1.00 & & 640 & 1.00 & & 403 & 1.00 & \\
\hline $5-29 \%$ poor & 1,901 & $1.95^{*}$ & $(0.94,4.03)$ & 507 & 1.02 & $(0.69,1.52)$ & 382 & 1.92 & $(1.02,3.61)$ \\
\hline$\geq 30 \%$ poor & 1,841 & 2.86 & $(1.40,5.82)$ & 382 & 1.37 & $(0.91,2.06)$ & 362 & 1.54 & $(0.83,2.84)$ \\
\hline \multicolumn{10}{|l|}{ Primary payer } \\
\hline Uninsured or Medicaid & 934 & 1.00 & & 224 & 1.00 & & 252 & 1.00 & \\
\hline Medicare or private & 4,783 & 1.13 & $(0.56,2.29)$ & 1,305 & 0.55 & $(0.37,0.82)$ & 894 & 0.65 & $(0.42,1.00)$ \\
\hline \multirow[t]{4}{*}{ Poverty by payer } & 5,717 & $0.52^{*}$ & $(0.25,1.11)$ & 1,529 & $1.03^{\mathrm{a}}$ & $(0.64,1.67)$ & 1,146 & $1.96^{*}$ & $(0.97,3.97)$ \\
\hline & \multicolumn{9}{|c|}{ Poverty by payer interaction on surgical wait times of more than 2 months } \\
\hline & & \multicolumn{3}{|c|}{$>5 \%$ poor } & & \multicolumn{3}{|c|}{$<5 \%$ poor } & \\
\hline & & Sample & OR & $(95 \% \mathrm{Cl})$ & & Sample & OR & $(95 \% \mathrm{Cl})$ & \\
\hline \multicolumn{10}{|l|}{ Primary payer } \\
\hline Uninsured or Medicaid & & 765 & 1.00 & & & 169 & 1.00 & & \\
\hline Medicare or private & & 2,977 & 0.58 & $(0.44,0.75)$ & & 1,806 & 1.18 & $(0.58,2.39)$ & \\
\hline
\end{tabular}

Poverty by payer interaction among women who received chemotherapy on post-surgical wait times of more than 7 months for RT

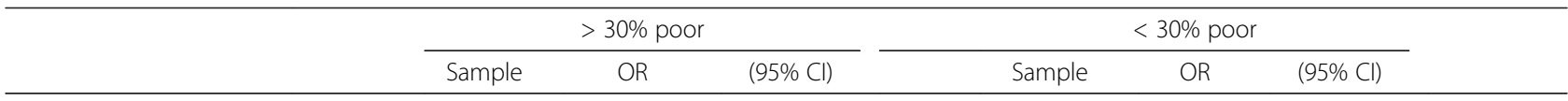

Primary payer

$\begin{array}{lllllll}\text { Uninsured or Medicaid } & 121 & 1.00 & & 131 & 1.00 \\ \text { Medicare or private } & 240 & 0.96 & (0.59,1.57) & 654 & 0.70^{*} & (0.47,1.03)\end{array}$

Notes. $O R=$ odds ratio, $C l=$ confidence interval. All effects were age and stage-adjusted across these categories: 25-44, 45-54, 55-64, 65-74 and 75 or older; and node positive or node negative breast cancer. Bolded ORs were statistically significant at $p<.05$.

${ }^{a}$ Null interaction was removed from the full model. ${ }^{*} p<10$.

The rate of waiting for more than seven months after surgery for radiation therapy among those covered by Medicare or private insurers $(16.2 \%)$ was approximately $25 \%$ lower than that of the uninsured or Medicaid covered $(21.8 \%, \mathrm{RR}=0.74,95 \%$ CI $0.52,1.06, p<.10)$.

Surgical wait times fit a different pattern. First, there was a very large effect of poverty $(\mathrm{OR}=2.86)$. Women who lived in high poverty neighborhoods (12.7\%) were two and a half times as likely to have waited two months or more for surgery than were women who lived in low poverty neighborhoods $(5.0 \%, \mathrm{RR}=2.54,95 \% \mathrm{CI} 2.05,3.15)$. Second, in the context of such a large effect of poverty, it seems that having adequate health insurance confers a protective effect in high and middle poverty $(\mathrm{OR}=0.58)$, but not in low poverty neighborhoods. In higher poverty neighborhoods, adequately insured women were about a third less likely than were uninsured or Medicaid insured women to have waited two months or more. Such respective long wait rates were $6.9 \%$ and $10.5 \%$ $(R R=0.66,95 \%$ CI 0.52, 0.96). Such long surgical wait 
Table 7 Logistic regression main effects and interactions of neighborhood poverty and primary payer on receipt of initial and adjuvant therapies among women with node negative breast cancer

\begin{tabular}{|c|c|c|c|c|c|c|c|c|c|}
\hline \multirow[b]{2}{*}{ Predictor variables } & \multicolumn{3}{|c|}{ Radiation therapy $^{a}$} & \multicolumn{3}{|c|}{ Chemotherapy } & \multicolumn{3}{|c|}{ Hormone therapy ${ }^{b}$} \\
\hline & Sample & OR & $(95 \% \mathrm{Cl})$ & Sample & OR & $(95 \% \mathrm{Cl})$ & Sample & OR & $(95 \% \mathrm{Cl})$ \\
\hline & \multicolumn{9}{|c|}{ Single predictor models } \\
\hline \multicolumn{10}{|c|}{ Neighborhood poverty } \\
\hline$<5 \%$ poor & 877 & 1.00 & & 1,327 & 1.00 & & 932 & 1.00 & \\
\hline $5-29 \%$ poor & 762 & $0.80^{*}$ & $(0.63,1.01)$ & 1,232 & 1.18 & $(0.94,1.46)$ & 756 & 0.77 & $(0.63,0.93)$ \\
\hline$\geq 30 \%$ poor & 611 & 0.63 & $(0.49,0.81)$ & 1,113 & 0.96 & $(0.76,1.22)$ & 611 & 0.70 & $(0.56,0.86)$ \\
\hline \multicolumn{10}{|l|}{ Primary payer } \\
\hline All others & 980 & 1.00 & & 1,627 & 1.00 & & 1,002 & 1.00 & \\
\hline \multirow[t]{2}{*}{ Private } & 1,270 & 1.40 & $(1.12,1.75)$ & 2,045 & 1.00 & $(0.81,1.24)$ & 1,297 & 1.38 & $(1.14,1.66)$ \\
\hline & \multicolumn{9}{|c|}{ Full models } \\
\hline \multicolumn{10}{|c|}{ Neighborhood poverty } \\
\hline$<5 \%$ poor & 877 & 1.00 & & 1,327 & 1.00 & & 932 & 1.00 & \\
\hline $5-29 \%$ poor & 762 & 0.62 & $(0.44,0.87)$ & 1,232 & 1.16 & $(0.93,1.44)$ & 756 & 0.78 & $(0.64,0.95)$ \\
\hline$\geq 30 \%$ poor & 611 & 0.51 & $(0.37,0.72)$ & 1,113 & 0.77 & $(0.54,1.10)$ & 611 & 0.73 & $(0.59,0.90)$ \\
\hline \multicolumn{10}{|l|}{ Primary payer } \\
\hline All others & 980 & 1.00 & & 1,627 & 1.00 & & 1,002 & 1.00 & \\
\hline Private & 1,270 & 0.97 & $(0.68,1.40)$ & 2,045 & 0.87 & $(0.66,1.13)$ & 1,297 & 1.32 & $(1.09,1.60)$ \\
\hline \multirow[t]{4}{*}{ Poverty by payer } & 2,250 & 1.61 & $(1.05,2.46)$ & 3,672 & $1.43^{*}$ & $(0.93,2.19)$ & 2,299 & $0.98^{c}$ & $(0.79,1.21)$ \\
\hline & \multicolumn{9}{|c|}{ Poverty by payer interaction on receipt of adjuvant radiation therapy } \\
\hline & & \multicolumn{3}{|c|}{$>5 \%$ poor } & \multicolumn{3}{|c|}{$<5 \%$ poor } & & \\
\hline & & Sample & OR & $(95 \% \mathrm{Cl})$ & Sample & OR & $(95 \% \mathrm{Cl})$ & & \\
\hline \multicolumn{10}{|l|}{ Primary payer } \\
\hline All others & & 651 & 1.00 & & 329 & 1.00 & & & \\
\hline \multirow[t]{4}{*}{ Private } & & 722 & 1.56 & $(1.19,2.03)$ & 548 & 1.06 & $(0.70,1.61)$ & & \\
\hline & \multicolumn{9}{|c|}{ Poverty by payer interaction on receipt of chemotherapy } \\
\hline & & \multicolumn{3}{|c|}{$>30 \%$ poor } & \multicolumn{3}{|c|}{$<30 \%$ poor } & & \\
\hline & & Sample & OR & $(95 \% \mathrm{Cl})$ & Sample & OR & $(95 \% \mathrm{Cl})$ & & \\
\hline \multicolumn{10}{|l|}{ Primary payer } \\
\hline All others & & 639 & 1.00 & & 988 & 1.00 & & & \\
\hline Private & & 474 & $1.39^{*}$ & $(0.94,2.06)$ & 1,571 & 0.86 & $(0.65,1.13)$ & & \\
\hline
\end{tabular}

Notes. $O R=$ odds ratio, $C l=$ confidence interval. All effects were age and tumor size-adjusted across these categories: 25-44, 45-54, 55-64, 65-74 and 75 or older; and less than 10, 10-19 and 20-50 mm or larger. Bolded ORs were statistically significant at $p<.05$.

${ }^{\mathrm{a}}$ Among women who received breast conserving surgery. ${ }^{\mathrm{b}}$ Among women with hormone receptor positive tumors.

c Null interaction was removed from the full model. ${ }^{*} p<.10$.

rates were identical (5.5\%) for both payer groups in low poverty neighborhoods.

\section{Receipt of adjuvant therapies}

The effects of poverty and payer on the receipt of adjuvant therapies for node negative and node positive breast cancers are, respectively, displayed in Tables 7 and 8 . It should be noted that nearly all of them received initial surgery (98.5\%) and such receipt was not associated with poverty or any payers. For women with node negative disease having private health insurance seemed to make the biggest difference and for women with node positive disease having any form of health insurance seemed to confer substantial access advantages over the uninsured. For women with node negative disease, in addition to having large main disadvantageous effects on access to radiation and hormone therapies, poverty seemed to moderate the effect that private insurance had in providing access to both adjuvant radiation and chemotherapies. Their poverty by payer interactions were, respectively, significant or nearly significant. The interactions depicted in the bottom of the table indicated protective effects conferred by having private health insurance specific to higher poverty neighborhoods. In high to middle poverty neighborhoods more than three-quarters of the women who had received breast conserving surgery and 
Table 8 Logistic regression main effects and interactions of neighborhood poverty and primary payer on receipt of initial and adjuvant therapies among women with non-distally metastasized, node positive breast cancer

\begin{tabular}{|c|c|c|c|c|c|c|c|c|c|}
\hline \multirow[b]{2}{*}{ Predictor variables } & \multicolumn{3}{|c|}{ Radiation therapy } & \multicolumn{3}{|c|}{ Chemotherapy $^{a}$} & \multicolumn{3}{|c|}{ Hormone therapy ${ }^{b}$} \\
\hline & Sample & OR & $(95 \% \mathrm{Cl})$ & Sample & OR & $(95 \% \mathrm{Cl})$ & Sample & OR & $(95 \% \mathrm{Cl})$ \\
\hline & \multicolumn{9}{|c|}{ Single predictor models } \\
\hline \multicolumn{10}{|l|}{ Neighborhood poverty } \\
\hline$<5 \%$ poor & 497 & 1.00 & & 364 & 1.00 & & 364 & 1.00 & \\
\hline $5-29 \%$ poor & 525 & 0.82 & $(0.64,1.05)$ & 321 & 1.01 & $(0.73,1.40)$ & 333 & 1.19 & $(0.88,1.62)$ \\
\hline$\geq 30 \%$ poor & 568 & 0.75 & $(0.59,0.96)$ & 393 & 0.78 & $(0.56,1.07)$ & 335 & 0.90 & $(0.66,1.23)$ \\
\hline \multicolumn{10}{|l|}{ Primary payer } \\
\hline Uninsured & 174 & 1.00 & & 107 & 1.00 & & 103 & 1.00 & \\
\hline \multirow[t]{2}{*}{ Any insurance } & 1,416 & 0.94 & $(0.76,1.16)$ & 931 & 1.12 & $(0.72,1.75)$ & 929 & 0.98 & $(0.64,1.50)$ \\
\hline & \multicolumn{9}{|c|}{ Full models } \\
\hline \multicolumn{10}{|l|}{ Neighborhood poverty } \\
\hline$<5 \%$ poor & 497 & 1.00 & & 324 & 1.00 & & 364 & 1.00 & \\
\hline $5-29 \%$ poor & 525 & 0.82 & $(0.63,1.05)$ & 321 & 1.07 & $(0.77,1.49)$ & 333 & $1.32^{*}$ & $(0.96,1.82)$ \\
\hline$\geq 30 \%$ poor & 568 & 0.73 & $(0.57,0.93)$ & 393 & 0.86 & $(0.62,1.21)$ & 335 & 0.90 & $(0.66,1.22)$ \\
\hline \multicolumn{10}{|l|}{ Primary payer } \\
\hline Uninsured & 174 & 1.00 & & 107 & 1.00 & & 103 & 1.00 & \\
\hline Any insurance & 1,416 & 0.87 & $(0.70,1.09)$ & 931 & 0.64 & $(0.32,1.29)$ & 929 & 0.68 & $(0.41,1.15)$ \\
\hline \multirow[t]{4}{*}{ Poverty by payer } & 1,590 & $0.73^{c}$ & $(0.48,1.12)$ & 1,038 & $0.59^{*}$ & $(0.34,1.01)$ & 1,032 & 0.33 & $(0.13,0.82)$ \\
\hline & \multicolumn{9}{|c|}{ Poverty by payer interaction on receipt of chemotherapy } \\
\hline & & \multicolumn{3}{|c|}{$>5 \%$ poor } & & \multicolumn{3}{|c|}{$<5 \%$ poor } & \\
\hline & & Sample & OR & $(95 \% \mathrm{Cl})$ & & Sample & OR & $(95 \% \mathrm{Cl})$ & \\
\hline \multicolumn{10}{|l|}{ Primary payer } \\
\hline Uninsured & & 75 & 1.00 & & & 32 & 1.00 & & \\
\hline \multirow[t]{4}{*}{ Any insurance } & & 639 & $1.63^{*}$ & $(0.91,2.90)$ & & 292 & 0.55 & $(0.25,1.17)$ & \\
\hline & \multicolumn{9}{|c|}{ Poverty by payer interaction on receipt of hormone therapy } \\
\hline & \multicolumn{3}{|c|}{$>30 \%$ poor } & \multicolumn{3}{|c|}{$5-29 \%$ poor } & \multicolumn{3}{|c|}{$<5 \%$ poor } \\
\hline & Sample & $\mathrm{OR}$ & $(95 \% \mathrm{Cl})$ & Sample & $\mathrm{OR}$ & $(95 \% \mathrm{Cl})$ & Sample & $\mathrm{OR}$ & $(95 \% \mathrm{Cl})$ \\
\hline \multicolumn{10}{|l|}{ Primary payer } \\
\hline Uninsured & 36 & 1.00 & & 32 & 1.00 & & 35 & 1.00 & \\
\hline Any insurance & 299 & 0.72 & $(0.34,1.52)$ & 301 & 2.16 & $(1.00,4.69)$ & 329 & 0.59 & $(0.28,1.26)$ \\
\hline
\end{tabular}

Notes. $\mathrm{OR}=$ odds ratio, $\mathrm{Cl}=$ confidence interval. Effects on radiation and hormone therapies were age and tumor size-adjusted and effects on chemotherapy were adjusted for age and the receipt of radiation therapy across these categories: $25-44,45-54,55-64,65-74$ and 75 or older; less than 10 , $10-19$ and $20-50$ mm or larger; and received radiation therapy or not. Bolded ORs were statistically significant at $p<.05$.

${ }^{a}$ Among women who received mastectomies. ${ }^{b}$ Among women with hormone receptor positive tumors.

${ }^{c}$ Null interaction was removed from the full model. ${ }^{*} p<.10$.

were privately insured received adjuvant radiation therapy (77.3\%), whereas, only about two-thirds of otherwise similar women who were covered by Medicare, Medicaid or were uninsured did $(67.6 \%, \mathrm{RR}=1.15$, $95 \% 1.07,1.23)$. No such effect was observed in low poverty neighborhoods. In high poverty neighborhoods there was an even larger effect of being privately insured on chemotherapy receipt. Respectively, 24.6\% and $19.6 \%$ of privately insured and other insured or uninsured women received chemotherapy ( $R R=1.26,95 \%$ $1.00,1.58)$. There was no significant effect of being privately insured in lower poverty neighborhoods. As for hormone therapy, only significant main effects of poverty and private insurance were observed. Again, having private insurance or not made a big difference in access to hormone therapy $(50.7 \%$ versus $41.7 \%, R R=1.22$, 95\% CI 1.12, 1.33).

For women with node positive disease having any form of health insurance seemed to confer substantial advantages over the uninsured. Poverty again moderated the effects of these payers on access to chemotherapy and hormone therapy (middle and right columns of Table 8). Large care gaps were observed among the uninsured, particularly among the 
uninsured that lived in the poorest neighborhoods. Such residents of high poverty neighborhoods who had any health insurance were much more likely to have received chemotherapy $(85.5 \%$ vs $65.4 \%, \mathrm{RR}=$ $1.31,95 \%$ CI 1.16, 1.48). And such insured residents of middle poverty neighborhoods seemed more likely to have received hormone therapy $(53.3 \%$ versus $36.6 \%, \mathrm{RR}=1.46,95 \%$ CI $0.94,2.27, p<.10)$. Not surprisingly, as it is not typically the first adjuvant treatment choice for node positive breast cancer, no significant payer effect was observed for radiation therapy.

\section{Specialized care among women with non-metastasized disease}

Next, we examined surgical procedures that came into practice (sentinel lymph node biopsy [SLNB]) or continued to proliferate during the study period (breast conserving surgery $[\mathrm{BCS}]$ ) or that are not yet prevalently provided (breast reconstruction surgery [BRS]. There were similar patterns of Medicaid main effects and poverty by private insurance interactions for BCS and BRS. Those with Medicaid were less likely to receive BCS (37.1\% vs $50.4 \%, \mathrm{RR}=0.74,95 \% \mathrm{CI} 0.65,0.85)$ and much less likely to receive BRS ( $3.2 \%$ vs $13.7 \%, R R=0.23,95 \%$ CI $0.12,0.46)$ than other women. And private insurance facilitated access to both BCS (50.2\% vs $41.6 \%$, RR $=1.21$, $95 \%$ CI $1.07,1.36)$ and BRS $(12.9 \%$ vs $5.9 \%, \mathrm{RR}=2.19$, $95 \%$ CI $1.55,3.09$ ) in high and middle poverty neighborhoods, but not in low poverty ones. Medicare or private health insurance completely mediated the disadvantaging effect of poverty on receipt of SLNB. Moreover, such coverage seemed to greatly facilitate access in low poverty neighborhoods $(14.8 \%$ vs $6.6 \%, \mathrm{RR}=2.24,95 \%$ CI $1.19,4.20)$ more modestly facilitate access in middle poverty neighborhoods $(10.8 \%$ vs $6.2 \%, \mathrm{RR}=1.74,95 \% \mathrm{CI} 1.04,2.91)$, but seemed to have no effect in high poverty neighborhoods were very few women $(6.3 \%)$ received such an innovative procedure.

\section{Explorations of palliative care among women with metastasized disease}

There seemed to be main effects of health insurance, but not poverty on the receipt of surgery and chemotherapy. Those with any insurance were about 50\% more likely than the uninsured to have received surgery (46.7\% vs $31.7 \%, \mathrm{RR}=1.47,95 \%$ CI $0.94,2.29, p<.10)$ and those with Medicare or private coverage were $25 \%$ more likely to have received chemotherapy than the uninsured or Medicaid covered (58.8\% vs $47.2 \%, \mathrm{RR}=1.25$, $95 \%$ CI $0.98,1.60, p<.10)$. A protective effect of private insurance on the receipt of radiation therapy was only observed in middle poverty neighborhoods. More than half of such privately insured women received radiation therapy (52.6\%), whereas only about a third of those covered by other than commercial insurers or who were uninsured did (35.3\%, RR $=1.49,95 \%$ CI 0.95, 2.35, $p<.10)$. No significant main or interacting effects of health insurance were observed on hormone therapy. There was, however, quite a strong effect of poverty. Women in high poverty neighborhoods were only about half as likely to receive hormone therapy as were their counterparts in low poverty neighborhoods $(36.4 \%$ vs $69.2 \%, \mathrm{RR}=0.53,95 \%$ CI $0.33,0.84)$.

\section{Discussion}

We found strong support for our hypothesis that health insurance mediates the poverty-breast cancer survival relationship. Evidence of survival mediation was observed for women with the most common and treatable type of breast cancer, node negative. The effect of poverty disappeared in the presence of Medicare or private insurance. Women who were so insured were advantaged on survival compared to the uninsured or those insured by Medicaid. Evidence of insurance effect moderation by poverty was also observed for women with node negative disease. The survival advantaging effect of Medicare or private insurance was strongest in low poverty neighborhoods, less strong in moderately poor neighborhoods and nonexistent in high poverty neighborhoods. This same pattern of mediated and moderated effects was also observed for stage at diagnosis, waits for adjuvant radiation therapy and for receipt of sentinel lymph node biopsies. These findings are consistent with the theory that more facilitative social and economic capital is available in more affluent neighborhoods where women with breast cancer may be better able to absorb the indirect and direct, but uncovered, costs of care.

We did not find support for our hypothesis that Medicaid would also mediate the effect of poverty as we had for women with colon cancer [27]. However, this study's sample of women with breast cancer and the previous study's sample of women with colon cancer were demographically distinct. The women with breast cancer were much younger. They were twice as likely to be of pre-retirement age and 35\% more likely to be covered by a commercial insurer. Younger women with breast cancer, more than half of whom had private health insurance, benefited greatly from having it. Whereas, older women with colon cancer, two-thirds of whom did not have private insurance, seemed to benefit relatively more from Medicaid coverage.

Medicare or private insurers were the primary payers for the care of eight out of every ten women in this study. It seems that the effectiveness of these, most prevalent, health insurance programs, public and private, are significantly impacted by the availability of other 
resources. In more well to do neighborhoods where social and economic capital abounds most women with breast cancer seem able to absorb the uncovered costs of cancer care. But high poverty neighborhoods, with their relative lack of such capital reserves, seem to remain places of "true disadvantage" [6], especially for the women who live there. Not only are they much more likely to be uninsured or underinsured, but even when insured such insurance programs seem to be less effective for them there than they are in less impoverished neighborhoods.

As for treatments, main and moderator effects indicative of protection, particularly in high poverty neighborhoods were observed for women with private health insurance. All of the following benefits of having commercial coverage among women living in high poverty neighborhoods were observed: shorter surgical waits, better access to BCS as well as to adjuvant radiation, chemo- and hormone therapies. And for women who received mastectomies, those with private insurance were more than twice as likely to have also received BRS.

These findings may seem counter hypothetical, but an extension of our health insurance-social capital theory could explain them. In such high poverty neighborhoods, having private health insurance could itself be deemed an important form of social capital. Especially in such vulnerable places, private health insurance seems to operate to the advantage of its holders. One way that it may operate is through its probable association with higher quality primary care. Privately insured women are more likely to have more established, continuous relationships with primary care physicians. Such relationships are known to provide myriad benefits: preventive surveillance, referral to and follow-up of specialist care, and advocacy and coordination throughout care processes $[60,61]$. Clearly, such could make a very big difference in the life of a woman with breast cancer, especially if she otherwise has limited personal and community resources available to her. This is not to say that high poverty neighborhoods are devoid of such resources. For example, evidence has been accumulating about the possible health benefits of living in certain relatively homogeneous communities such as Mexican American barrios $[62,63]$. Though such ethnic concentrations tend to be associated with concentrations of poverty, it seems that people and institutions in these communities provide quite a bit of effective social and economic support to one another. Despite their resiliencies though, gaps in access to key resources including primary care have been identified there. So it stands to reason that private insurance and attendant primary care could operate to potentiate the strengths and resiliencies that already exist in such high poverty neighborhoods.

\section{Payer effect sizes}

This study's payer effect sizes estimated with standardized rate ratios ranged from 1.06 to 2.25. Those most hypothetically central, the effects of having Medicare or private insurance on early diagnosis and survival among women with node negative breast cancer in high poverty neighborhoods, ranged from 1.10 to 1.19 . Though significant in a statistical sense, they might be thought small for clinical practice or policy guidance. We think such would be an interpretive error for the following reasons. The attribution of risk at the population level is a function of three factors of which effect size is only one. It is also important to consider the size of the population at risk as well as the prevalence of exposures to the risk factors being studied. In this instance, the central exposure or risk factor to be mediated is a social one, that is, residence in a high poverty neighborhood. The other social exposure of interest or risk factor is the risk of being inadequately insured, that is, of being insured by Medicaid or uninsured. More than forty percent of the non-elderly, non-disabled California population or more than 13 million people are so affected. The national estimate approaches 100 million [64-66]. At the time of this study approximately 34 million Americans were poor and nearly 10 million of them lived in high poverty neighborhoods $[35,67]$. Regrettably, being uninsured, Medicaid insured or poor are not rare "exposures" in California or America. And nearly a quarter of a million American women are newly diagnosed with breast cancer each year [68]. So a change in relative risk of ten to twenty percent could affect more than 10 thousand women with breast cancer in California and nearly 50 thousand women in the United States each year. In terms of population attributable risk, we would deem these extraordinarily large effects.

\section{Potential limitations}

Our use of ecological poverty measures might suggest alternative explanations for our results. One may wonder if the racial/ethnic composition of high poverty neighborhoods, rather than their concentration of the poor, accounted for the care and survival differences we observed. We do not think so for the following reasons. First, numerous recent US studies of breast cancer care and survival have found that socioeconomic differences explain most racial-group differences [69-74]. Second, after we accounted for age, and stage as well as the main and interacting effects of poverty and health insurance in these analyses, race/ethnicity was not significantly associated with survival. And third, our research group observed that health insurance disparities can explain essentially all of the breast cancer care disparities observed among our sample's largest ethnic minority group (15.7\%), Mexican Americans [75,76]. In short, while not 
refuting the importance of race, ethnicity and culture in cancer care [77-79], our analyses suggest that having adequate health insurance is probably much more important.

There has been a large unmet need for research on the validity of ecological measures of SES often used in public health research. Wilson and Jargowsky and others have added much knowledge on high poverty neighborhood measures [6-8,35,36] and our research group has done work to advance understandings of poor neighborhoods, analogous to this study's middle poverty neighborhoods $[80,81]$. They seem prevalently represented by not only the poor, but the near poor and working poor as well as lower-middle and middle class people. This study added further to our knowledge about vulnerable neighborhoods. For example, steep gradients were observed for various types of health insurance across low (e.g., baseline Medicaid coverage) to high poverty neighborhoods (prevalence more than 12 -fold greater [82,83]). This new knowledge about the validity of ecological poverty measures may advance our understandings about the contexts in which very poor Americans live. Therefore, our findings are probably not prone to ecological fallacy. They may, in fact, help researchers to better understand contextual measures of SES and so avoid individualistic fallacies of inference $[84,85]$.

Another possible limitation of our study was incomplete information on outpatient treatments $[51,86]$. Such data are more difficult for cancer registries to collect than inpatient data. However, the California breast cancer registry has been enhanced well beyond the norm and has been shown to be nearly complete for chemotherapy data [34,87]. In addition, analyses of disease stage, hospital-based surgery and survival were unlikely to have been affected [88], and missing radiation therapy and chemotherapy data were not prevalent in our sample. Finally, we focused on overall survival, rather than cancer-specific survival. Although vital status and length of overall survival are highly accurate in cancer registries, the underlying cause of death probably is not [89-91]. Although death certificate error was a likely limitation, we did systematically replicate our central all-cause survival hypothesis tests with cancer-specific ones. If anything, our overall survival effects were slight underestimates of cancer-specific ones [90]. And the underlying cause of many deaths not coded as cancer mortality can be directly associated with lack of treatment or with cancer treatment complications [92]. For all of these reasons, we think overall survival a valid and policytelling outcome. And such would clearly have had no impact on other indices of breast cancer care such as diagnoses and treatments.

\section{Conclusions}

America's multi-tiered health insurance system mediates the quality of breast cancer care. The system is inequitable and unjust as it advantages the well insured and the well to do. Recent health care reforms ought to be enacted across all 50 states in ways that are consistent with their federal legislative intent, that high quality health care be truly available to all.

\section{Human participants ethical review}

This study was reviewed and cleared by the University of Windsor's research ethics board.

\section{Competing interests}

The authors declare that they have no competing interests.

\section{Authors' contributions}

KMG conceptualized and supervised the study and led the writing. KMG, INL, $\mathrm{EJH}, \mathrm{GYZ}$ and $\mathrm{CH}$ obtained funding. GYZ supervised the analysis. All authors assisted with study design, data analysis and interpretation, and writing. All authors read and approved the final manuscript.

\section{Acknowledgements}

This research was supported in part with funds from the Canadian Institutes of Health Research (grant no. 67161). The authors gratefully acknowledge the administrative and logistical assistance of Kurt Snipes, Janet Bates and Gretchen Agha of the Cancer Surveillance and Research Branch, California Department of Public Health. We also gratefully acknowledge the research and technical assistance of Nancy Richter and Nazim Habibov of the University of Windsor and Mark Allen and Allyn Fernandez-Ami of the California Cancer Registry.

The collection of cancer incidence data used in this study was supported by the California Department of Public Health as part of the statewide cancer reporting program mandated by California Health and Safety Code Section 103885; the National Cancer Institute's Surveillance, Epidemiology and End Results Program under contract HHSN261201000140C, awarded to the Cancer Prevention Institute of California, contract HHSN261201000035C, awarded to the University of Southern California, and contract HHSN261201000034C, awarded to the Public Health Institute; and the Centers for Disease Control and Prevention's National Program of Cancer Registries, under agreement U58DP003862-01 awarded to the, California Department of Public Health. The ideas and opinions expressed herein are those of the authors and endorsement by the State of California,

Department of Public Health the National Cancer Institute, and the Centers for Disease Control and Prevention or their contractors and subcontractors are not intended nor should be inferred.

\section{Author details}

${ }^{1}$ School of Social Work, University of Windsor, 401 Sunset Avenue, Windsor, Ontario N9B 3P4, Canada. ²Department of Geography, University of Western Ontario, London, Ontario, Canada. ${ }^{3}$ Dalla Lana School of Public Health, University of Toronto, Toronto, Ontario, Canada. ${ }^{4}$ Department of Epidemiology and Biostatistics, University of Western Ontario and Scientist, Robarts Research Institute, London, Ontario, Canada. ${ }^{5}$ Medical Oncologist, Windsor Regional Cancer Center, School of Medicine and Dentistry, Department of Medicine, Division of General Internal Medicine, University of Western Ontario, London, Ontario, Canada. ${ }^{6}$ Statistician and Research Associate, School of Social Work, University of Windsor, Windsor, Ontario, Canada.

Received: 31 July 2012 Accepted: 7 January 2013

Published: 14 January 2013

\section{References}

1. Gorey KM, Holowaty EJ, Fehringer G, Laukkanen E, Webster DJ, Moskowitz A, Richter NL: An international comparison of cancer survival: Toronto, Ontario, and Detroit, Michigan, metropolitan areas. Am J Public Health 1997, 87:1156-1163. 
2. Gorey KM: Breast cancer survival in Canada and the United States: metaanalytic evidence of a Canadian advantage in low-income areas. Int J Epidemiol 2009, 38:1543-1551.

3. Gorey KM, Luginaah IN, Holowaty EJ, Fung KY, Hamm C: Wait times for surgical and adjuvant radiation treatment of breast cancer in Canada and the United States: greater socioeconomic inequity in America. Clin Invest Med 2009, 32:E239-E249.

4. Gorey KM, Fung KY, Luginaah IN, Holowaty EJ, Hamm C: Income and longterm breast cancer survival: comparisons of vulnerable urban places in Ontario and California. Breast J 2010, 16:416-419.

5. Gorey KM, Luginaah IN, Hamm C, Fung KY, Holowaty EJ: Breast cancer care in Canada and the United States: ecological comparisons of extremely impoverished and affluent urban neighborhoods. Health Place 2010, 16:156-163.

6. Wilson WJ: The truly disadvantaged: the inner city, the underclass, and public policy. University of Chicago Press; 1987.

7. Jargowsky PA: Poverty and place: ghettos, barrios, and the American city. New York: Russell Sage Foundation; 1997.

8. Jargowsky PA, Bane MJ: Ghetto poverty in the United States, 1970-1980. In The urban underclass (pp. 235-273). Edited by Jencks C, Peterson PE. Washington, DC: The Brookings Institution; 1991.

9. Ries LAG, Eisner MP, Kosary CL, Hankey BF, Miller BA, Clegg L, Edwards BK (Eds): SEER cancer statistics review, 1975-2005. Bethesda, MD: National Cancer Institute; 2008.

10. Coleman MP, Quaresma M, Berrino F, Lutz JM, Capocaccia R, Baili P, Rachet B, Gatta G, Hakulinen T, Micheli A, Sant M, Weir HK, Elwood JM, Tsukuma H, Koifman SE, Silva GA, Francisci S, Santaquilani M, Verdecchia A, Storm HH, Young $J$, CONCORD Working Group: Cancer survival in five continents: a worldwide population-based study. Lancet Oncol 2008, 9:730-756

11. McKenzie F, Jeffreys M: Do lifestyle or social factors explain ethnic/racial inequalities in breast cancer survival? Epidemiol Rev 2009, 31:52-66.

12. Sabatino SA, Thompson TD, Richardson LC, Miller J: Health insurance and other factors associated with mammography surveillance among breast cancer survivors: results from a national survey. Med Care 2012, 50:270-276.

13. Hahn KME, Bondy ML, Selvan M, Lund MJ, Liff JM, Flagg EW, Brinton LA, Porter P, Eley JW, Coates RJ: Factors associated with advanced disease stage at diagnosis in a population-based study of patients with newly diagnosed breast cancer. Am J Epidemiol 2007, 166:1035-1044.

14. Gong G, Belasco E, Hargrave KA, Lyford CP, Philips BU: Determinants of delayed detection of cancers in Texas counties in the United States of America. Int J Equity Health 2012, 11:29.

15. Wu X-C, Lund MJ, Kimmick GG, Richardson LC, Sabatino SA, Chen WW, Fleming ST, Morris CR, Huang B, Trentham-Dietz A, Lipscomb J: Influence of race, insurance, socioeconomic status, and hospital type on receipt of guideline-concordant adjuvant systemic therapy for locoregional breast cancers. J Clin Oncol 2012, 30:142-150.

16. Yen TWF, Czypinski LK, Sparapani RA, Guo C, Laud PW, Pezzin LE, Nattinger $A B$ : Socioeconomic factors associated with adjuvant hormone therapy use in older breast cancer survivors. Cancer 2011, 117:398-405.

17. Bigby JA, Holmes MD: Disparities across the breast cancer continuum. Cancer Causes Control 2005, 16:35-44.

18. Shi L, Lebrun LA, Zhu J, Tsai MJ: Cancer screening among racial/ethnic and insurance groups in the United States: a comparison of disparities in 2000 and 2008. J Health Care Poor Underserved 2011, 22:945-961.

19. Schueler KM, Chu PW, Smith-Bindman R: Factors associated with mammography utilization: a systematic quantitative review of the literature. J Womens Health 2008, 17:1477-1498.

20. DeSantis C, Jemal A, Ward E: Disparities in breast cancer prognostic factors by race, insurance status, and education. Cancer Causes Control 2010, 21:1445-1450.

21. Kuzmiak CM, Haberle S, Padungchaichote W, Zeng D, Cole E, Pisano ED: Insurance status and the severity of breast cancer at the time of diagnosis. Acad Radiol 2008, 15:1255-1258.

22. Royak-Schaler R, Pelser C, Langenberg P, Hayes J, Gardner L, Nesbitt K, Citron W, Drogula CL, Dwyer D: Characteristics associated with the initiation of radiation therapy after breast-conserving surgery among African American and white women diagnosed with early-stage breast cancer in Maryland, 2000-2006. Ann Epidemiol 2012, 22:28-36.

23. Kruper L, Holt A, Xu XX, Duan L, Henderson K, Bernstein L, Ellenhorn J: Disparities in reconstruction rates after mastectomy: patterns of care and factors associated with the use of breast reconstruction in southern California. Ann Surg Oncol 2011, 18:2158-2165.

24. Dragun AE, Huang B, Tucker TC, Spanos WJ: Disparities in the application of adjuvant radiotherapy after breast-conserving surgery for early stage breast cancer: impact on overall survival. Cancer 2011, 117:2590-2598.

25. Gorey KM, Luginaah IN, Schwartz KL, Fung KY, Balagurusamy M, Bartfay E, Wright FC, Anucha U, Parsons RR: Increased racial differences on breast cancer care and survival in America: Historical evidence consistent with a health insurance hypothesis, 1975 to 2001. Breast Cancer Res Treat 2009,

113:595-600.

26. Gorey KM, Luginaah IN, Bartfay E, Fung KY, Holowaty EJ, Wright FC, Hamm C, Kanjeekal SM: Effects of socioeconomic status on colon cancer treatment accessibility and survival in Toronto, Ontario, and San Francisco, California, 1996-2006. Am J Public Health 2011, 101:112-119.

27. Gorey KM, Luginaah IN, Holowaty EJ, Zou G, Hamm C, Bartfay E, Kanjeekal SM, Balagurusamy MK, Haji-Jama S, Wright FC: Effects of being uninsured or underinsured and living in high poverty neighborhoods on colon cancer care and survival in California: Historical cohort analysis, 1996-2011. BMC Public Health 2012, 12:897.

28. Shankaran V, Jolly S, Blough D, Ramsey SD: Risk factors for financial hardship in patients receiving adjuvant chemotherapy for colon cancer: a population-based exploratory analysis. J Clin Oncol 2012, 30:1608-1614.

29. Sedjo RL, Devine S: Predictors of non-adherence to aromatase inhibitors among commercially insured women with breast cancer. Breast Cancer Res Treat 2011, 125:191-200.

30. Mackinnon DP: Integrating mediators and moderators in research design. Res Social Work Prac 2011, 21:675-681.

31. Greenland S, Rothman KJ: Introduction to stratified analysis. In Modern epidemiology, $2^{\text {nd }}$ ed (pp 253-279). Edited by Rothman KJ, Greenland S. Philadelphia, PA: Lippincott-Raven Publishers; 1998.

32. North American Association of Central Cancer Registries: Data quality assessments, 2008. Available at: http://www.naaccr.org. Accessed May 12, 2012

33. National Cancer Institute: Surveillance, epidemiology, and end results (SEER), 2008. Available at: http://www.seer.cancer.gov. Accessed May 12, 2012.

34. Wright WE: California cancer registry enhancement for breast cancer research. Berkeley: California Public Health Foundation; 1996.

35. Jargowsky PA: Stunning progress, hidden problems: the dramatic decline of concentrated poverty in the 1990s. In Redefining urban and suburban America: evidence from census 2000, Vol 2 (pp 137-171). Edited by Berube A, Katz B, Lang RE. Washington, DC: Brookings Institution Press; 2005.

36. Berube A, Frey $\mathrm{WH}$ : A decade of mixed blessings: urban and suburban poverty in census 2000. In Redefining urban and suburban America: evidence from census 2000, Vol 2 (pp 111-136). Edited by Berube A, Katz B, Lang RE. Washington, DC: Brookings Institution Press; 2005.

37. International Classification of Diseases: Ninth Revision. Geneva, Switzerland: World Health Organization; 1980

38. 2000 Census of population and housing in California: summary tape file 3 on CD-ROM. Washington, DC: US Census Bureau; 2002

39. Shugarman LR, Sorbero MES, Tian H, Jain AK, Ashwood JS: An exploration of urban and rural differences in lung cancer survival among Medicare beneficiaries. Am J Public Health 2008, 98:1280-1287.

40. Statistics Canada: Definitions of "rural". Ottawa: 2002

41. Fleiss JL, Levin B, Paik MC: Statistical methods for rates and proportions. 3rd edition. Hoboken, NJ: Wiley; 2003.

42. Faul F, Erdfelder E, Lang A-G, Buchner A: G*Power 3: a flexible statistical power analysis program for the social, behavioral, and biomedical sciences. Behav Res Meth 2007, 39:175-191.

43. Krieger N, Chen JT, Waterman PD, Rehkopf DH, Subramanian SV: Race/ ethnicity, gender, and monitoring socioeconomic gradients in health: a comparison of area based socioeconomic measures-the Public Health Disparities Geocoding Project. Am J Public Health 2003, 93:1655-1671.

44. Krieger N, Chen JT, Waterman PD, Soobader M, Subramanian SV, Carson R: Geocoding and monitoring of US socioeconomic inequalities in mortality and cancer incidence: does the choice of area-based measure and geographic level matter? The Public Health Disparities Geocoding Project. Am J Epidemiol 2002, 156:471-482.

45. Lee BA, Marlay M: The right side of the tracks: affluent neighborhoods in the metropolitan United States. Soc Sci Q 2007, 88:766-789.

46. California Cancer Registry: Cancer reporting in California: abstracting and coding procedures for hospitals. In California cancer reporting system 
standards, Vol 1 (7th ed). Sacramento, CA: Department of Health Services, Cancer Surveillance Section; 2003.

47. Fritz $A$, Ries $L$ (Eds): SEER extent of disease: codes and coding instructions. 3rd edition. Bethesda, MD: National Cancer Institute; 1998

48. Mosunjac M, Park J, Strauss A, Birdsong G, Du V, Rizzo M, Gabram SG, Lund $\mathrm{MJ}$ : Time to treatment for patients receiving BCS in a public and a private university hospital in Atlanta. Breast J 2012, 18:163-167.

49. Bilimoria KY, Ko CY, Tomlinson JS, Stewart AK, Talamonti MS, Hynes DL, Winchester DP, Bentrem DJ: Wait times for cancer surgery in the United States: trends and predictors of delays. Ann Surg 2011, 253:779-785.

50. Chen Z, King W, Pearcey R, Kerba M, Mackillop WJ: The relationship between waiting time for radiotherapy and clinical outcomes: a systematic review of the literature. Radiother Oncol 2008, 87:3-16.

51. Lund MJ, Brawley OP, Ward KC, Young JL, Gabram SSG, Eley JW: Parity and disparity in first course treatment of invasive breast cancer. Breast Cancer Res Treat 2008, 109:545-557.

52. Hershman DL, Wang X, McBride R, Jacobson JS, Grann VR, Neugut Al: Delay in initiating adjuvant radiotherapy following breast conservation surgery and its impact on survival. Int J Radiat Oncol Biol Phys 2006, 65:1353-1360.

53. Greenland S: Introduction to regression modeling. In Modern Epidemiology, 2nd ed, (pp. 401-432). Edited by Rothman KJ, Greenland S. Philadelphia: Lippincott-Raven Publishers; 1998.

54. Gorey KM: Regarding "Associations between socioeconomic status and cancer survival.". Ann Epidemiol 2006, 16:789-791.

55. Hosmer DW, Lemeshow S: Applied logistic regression. 2nd edition. NY: John Wiley \& Sons; 2000.

56. Agresti A: Categorical data analysis. Hoboken: John Wiley \& Sons; 2002

57. Greenland S: Interpretation and choice of effect measures in epidemiologic analysis. Am J Epidemiol 1987, 125:761-768.

58. Mantel N, Haenszel W: Statistical aspects of the analysis of data from retrospective studies of disease. J Nat/ Cancer Inst 1959, 22:719-748.

59. Miettinen OS: Estimability and estimation in case referent-studies. Am J Epidemiol 1976, 103:226-235.

60. Starfield B: Commentary: How does 'insurance' improve equity in health? Int J Epidemiol 2009, 38:1551-1553.

61. Starfield B, Shi L, Macinko J: Contribution of primary care to health systems and health. Milbank Q 2005, 83:457-502.

62. Aranda MP, Ray LA, Snih SA, Ottenbacher KJ, Markides KS: The protective effect of neighborhood composition on increasing frailty among older Mexican Americans: A barrio advantage? J Aging Health 2011, 23:1189-1217

63. Eschbach K, Ostir GV, Patel KV, Markides KS, Goodwin JS: Neighborhood context and mortality among older Mexican Americans: Is there a barrio advantage? Am J Public Health 2004, 94:1807-1812.

64. Kaiser Family Foundation: The Kaiser Commission on Medicaid and the uninsured (pub no 8050-05). Washington, DC: 2012.

65. Foundation CHC: California health care almanac: California's uninsured. Oakland, CA: Employee Benefit Research Institute; 2010

66. Malin JL, Diamant AL, Leake B, Liu Y, Thind A, Kahn KL, Schneider EC, Epstein AM, Maly RC: Quality of care for breast cancer for uninsured women in California under the Breast and Cervical Cancer Prevention Treatment Act. J Clin Oncol 2010, 28:3479-3484.

67. Iceland J: Poverty in America. 3rd edition. Berkeley: University of California Press; 2013.

68. American Cancer Society: Breast cancer facts and figures, 2011-2012. Atlanta; 2012.

69. Purc-Stephenson RJ, Gorey KM: Lower adherence to screening mammography guidelines among ethnic minority women in America: a meta-analytic review. Prev Med 2008, 46:479-488.

70. Wells KJ, Roetzheim RG: Health disparities in receipt of screening mammography in Latinas: a critical review of recent literature. Cancer Control 2007, 14:369-379.

71. Du XL, Fang S, Meyer TE: Impact of treatment and socioeconomic status on racial disparities in survival among older women with breast cancer Am J Clin Oncol 2008, 31:125-132

72. Chu QD, Smith MH, Williams M, Panu L, Johnson LW, Shi R, Li BDL, Glass J: Race/ethnicity has no effect on outcome for breast cancer patients treated at an academic center with a public hospital. Cancer Epidemiol Biomarkers Prev 2009, 18:2157-2161.

73. Chagpar AB, Crutcher CR, Cornwell LB, McMasters KM: Primary tumor size, not race, determines outcomes in women with hormone-responsive breast cancer. Surgery 2011, 150:796-801.
74. Komenaka IK, Martinez ME, Pennington RE Jr, Hsu C-H, Clare SE, Thompson PA, Murphy C, Zork NM, Goulet RJ Jr: Race and ethnicity and breast cancer outcomes in an underinsured population. J Nat/ Cancer Inst 2010, 102:1178-1187.

75. Richter NL, Gorey KM, Haji-Jama S, Luginaah IN: Breast cancer care of Mexican American women with node negative disease: Historical cohort evidence of interacting barrio-health insurance advantages. BMC Womens Health, in press.

76. Haji-Jama S, Gorey KM, Luginaah IN, Balagurusamy MK, Hamm C: Health insurance mediation of the Mexican American non-Hispanic white disparity on early breast cancer diagnosis. Ethnic Health, in press.

77. Parise CA, Bauer KR, Caggiano V: Disparities in receipt of adjuvant radiation therapy after breast-conserving surgery among the cancerreporting regions of California. Cancer 2012, 118:2516-2524.

78. Albain KS, Unger JM, Crowley JJ, Coltman CA Jr, Hershman DL: Racial disparities in cancer survival among randomized clinical trials patients of the Southwest Oncology Group. J Natl Cancer Inst 2009, 101:984-992.

79. Bauer KR, Brown M, Cress RD, Parise CA, Caggiano V: Descriptive analysis of estrogen receptor (ER)-negative, progesterone receptor (PR)-negative, and HER2-negative invasive breast cancer, the so-called triple-negative phenotype: A population-based study from the California Cancer Registry. Cancer 2007, 109:1721-1728.

80. Gorey KM, Holowaty EJ, Fehringer G, Laukkanen E, Richter NL, Meyer CM: An international comparison of cancer survival: Toronto, Ontario and Honolulu. Hawaii. Am J Public Health 2000, 90:1866-1872.

81. Gorey KM, Kliewer E, Holowaty EJ, Laukkanen E, Ng EY: An international comparison of breast cancer survival: Winnipeg, Manitoba and Des Moines, lowa metropolitan areas. Ann Epidemiol 2003, 13:32-41.

82. Verrill C: Assessing the reliability and validity of primary payer information in central cancer registry data. In Paper presented at the annual meeting of the North American Association of Central Cancer Registries. Quebec City: June, 2010.

83. Chan JK, Gomez SL, O'Malley CD, Perkins Cl, Clarke CA: Validity of cancer registry Medicaid status against enrollment files: implications for population-based studies of cancer outcomes. Med Care 2008, 44:952-955.

84. Subramanian SV, Jones K, Kaddour A, Krieger N: Revisiting Robinson: the perils of individualistic and ecologic fallacy. Int J Epidemiol 2009, 38:342-360

85. Silver E: Race, neighborhood disadvantage, and violence among persons with mental disorders: the importance of contextual measurement. Law Hum Behav 2000, 24:449-456.

86. Malin JL, Kahn KL, Adams J, Kwan L, Laouri M, Ganz PA: Validity of cancer registry data for measuring the quality of breast cancer care. J Natl Cancer Inst 2002, 94:835-844.

87. Cress RD, Zaslavsky AM, West DW, Wolf RE, Felter MC, Ayanian JZ: Completeness of information on adjuvant therapies for colorectal cancer in population-based cancer registries. Med Care 2003, 41:1006-1012.

88. Cooper GS, Virnig B, Klabunde CN, Schussler N, Freeman J, Warren JL: Use of SEER-Medicare data for measuring cancer surgery. Med Care 2002, 40(8 Suppl):IV43-48.

89. Yin D, Morris CR, Bates JH, German RR: Effect of misclassified underlying cause of death on survival estimates of colon and rectal cancer. J Nat Cancer Inst 2011, 20(103):1130-1133.

90. Hsiao FY, Mullins CD, Onukwugha E, Pandya N, Hanna N: Comparative effectiveness of different chemotherapeutic regimens on survival of people aged 66 and older with stage III colon cancer: a "real world" analysis using Surveillance, Epidemiology, and End Results-Medicare data. J Am Geriatr Soc 2011, 59:1717-1723.

91. Lenfant C, Freidman L, Thom T: Fifty years of death certificates: the Framingham Heart Study. Ann Intern Med 1998, 129:1066-1067.

92. Brown BW, Brauner C, Minnotte MC: Noncancer deaths in White adult cancer patients. J Natl Cancer Inst 1993, 85:979-987.

doi:10.1186/1475-9276-12-6

Cite this article as: Gorey et al:: Mediation of the effects of living in extremely poor neighborhoods by health insurance: breast cancer care and survival in California, 1996 to 2011. International Journal for Equity in Health 2013 12:6. 